\title{
Modifying effect of Siraitia grosvenori extract on piperonyl butoxide-promoted hepatocarcinogenesis in rats
}

\author{
Hironobu Yasuno', Jihei Nishimura1,2, Yasuaki Dewa'1,2, Masako Muguruma1, \\ Masayoshi Takabatake ${ }^{1}$, Yuji Murata ${ }^{3}$, Makoto Shibutani ${ }^{1}$ and Kunitoshi Mitsumori ${ }^{1}$ \\ ${ }^{1}$ Laboratory of Veterinary Pathology, Tokyo University of Agriculture and Technology, Fuchu City, Tokyo 183-8509, \\ Japan. \\ ${ }^{2}$ United Graduate School of Veterinary Sciences, Gifu University, 1-1 Yanagida, Gifu City, Gifu 501-1193, Japan. \\ ${ }^{3}$ Biochemical Laboratory, Saraya Co., Ltd., Kashiwara, Osaka 582-0028, Japan.
}

(Received December 3, 2007; Accepted January 7, 2008)

\begin{abstract}
To examine the possible modifying effect of the extract of Siraitia grosvenori (SGE), a naturally occurring antioxidative agent, on piperonyl butoxide (PBO)-promoted hepatocarcinogenesis, male F344 rats were administered a single intraperitoneal injection of $N$-diethylnitrosamine (DEN) as an initiator followed by administration of a diet containing $2 \%$ PBO for 7 weeks with or without SGE $(1,000 \mathrm{ppm})$ in the drinking water. To enhance cellular proliferation, all animals underwent two-thirds partial hepatectomy 1 week after the commencement of PBO administration. Pretreatment with SGE was also applied to the PBO + SGE group for 2 weeks prior to DEN initiation. Liver immunohistochemistry revealed that although the PBO-mediated increase in the number of glutathione $S$-transferase placental form (GST-P)-positive foci and proliferating cell nuclear antigen-positive cells remained unaltered with SGE coadministration, the area of the GST-P-positive foci was increased. On the contrary, real-time RTPCR showed that coadministration of SGE increased hepatic GST and glutathione peroxidase (GSH-Px) antioxidant activities and mRNA expression levels of the phase II enzymes that are known to be transcriptionally up-regulated through the Nrf 2-Keap1-antioxidant responsive element (ARE) as well as the phase III enzymes. Furthermore, measurement of thiobarbituric acid-reactive substances showed a decrease in lipid peroxidation by SGE coadministration. The results suggest that SGE may exert hepatic antioxidant activity by up-regulating the genes under the control of the Nrf 2-Keap1-ARE transcriptional machinery; however, this activity was neither effective nor sufficient for suppression of PBO-promoted early hepatocarcinogenesis.
\end{abstract}

Key words: Piperonyl butoxide, Oxidative stress, Siraitia grosvenori, Antioxidant, Rat

\section{INTRODUCTION}

Piperonyl butoxide, $\alpha-[2-(2-$ butoxyethoxy)ethoxy]-4,5methylenedioxy-2-propyltoluene (PBO), a pesticide synergist, is widely used along with pyrethroids as a grain protector and domestic insecticide. $\mathrm{PBO}$ has been reported to act as a hepatocarcinogen in F344 rats fed on a $1.2 \%$ or $2.4 \%$ PBO-containing diet for 2 years (Takahashi et al., 1994 ). However, PBO has been shown to be negative in bacterial mutation assays, hypoxanthine-guanine phosphoribosyltransferase (HGPRT) assay, chromosomal aberrations test performed using Chinese hamster ovary cells, and in vitro unscheduled DNA synthesis test
(Beamand et al., 1996; Butler et al., 1996). In a two-stage rat hepatocarcinogenesis model, $\mathrm{PBO}$ has been reported to act as a liver tumor promoter in F344 rats (Okamiya et al., 1998). PBO acts as a mild cytochrome P450 (CYP) $1 \mathrm{~A} 1$ inducer by inefficient activation of the aryl hydrocarbon receptor (AhR). CYP1A1 is also known to play an important role in the chemical activation of xenobiotics to carcinogenic derivatives. Our previous study demonstrated that PBO has the potential to generate reactive oxygen species (ROS) by induction of CYP1A1 and suggested the possibility that DNA damage resulting from oxidative stresses due to ROS generation is involved in the mechanism of hepatocarcinogenesis by PBO in rats and mice

Correspondence: Hironobu Yasuno (E-mail: shiperfnoru@yahoo.co.jp) 
(Muguruma et al., 2007).

Siraitia grosvenori, a traditional Chinese fruit, belongs to the cucurbitaceous species and has been used as a folk medicine for sore throat, cough, and minor stomach and intestinal problems. It contains several sweet triterpene glycosides. Recently, the extract of Siraitia grosvenori (SGE) has been shown to exert a variety of physiological functions. For example, SGE reduces the atherogenic potential of low-density lipoprotein (LDL) by dose-dependent inhibition of copper-mediated oxidation and human umbilical vein endothelial cellmediated LDL oxidation (Takeo et al., 2001). SGE has also been shown to exert free radical scavenging activity (Shi et al., 1996) and to possess antiallergenic potential in a mouse model of histamine-induced nasal rubbing and compound 48/80-induced skin scratching (Hossen et al., 2005). Treatment of diabetic mice with SGE also prevented the development of diabetic nephropathy owing to its antioxidative action (Song et al., 2006). In addition, cucurbitane triterpene glycosides in SGE, namely, mogroside $\mathrm{V}$ and 11-oxo-mogroside $\mathrm{V}$, have been shown to inhibit the tumor-promoting activity of $12-O$ tetradecanoylphorbol-13-acetate in a two-stage mouse skin carcinogenesis model; they have also been shown to inhibit the tumor-initiating activity of peroxynitrite (Takasaki et al., 2003).

In view of the antioxidative properties of SGE, we, in the present study, investigated (1) the modifying effect of SGE on PBO-promoted hepatocarcinogenesis in rats and (2) the molecular events related to its antioxidative responses in a two-stage rat hepatocarcinogenesis model.

\section{MATERIALS AND METHODS}

\section{Chemical, animals, and treatment}

PBO (CAS 51-03-6; technical grade; purity, > 90\%) was purchased from ACROS Organics (Morris Plains, NJ, USA). SGE was kindly supplied by the biochemical laboratory Saraya Co., Ltd. (Osaka, Japan). A total of 36 male F344/N Slc rats aged 4 weeks and weighing $53.75 \pm 6.19$ $\mathrm{g}$ were purchased from Japan SLC Inc. (Shizuoka, Japan). They were assigned to the control, PBO-treated (PBO) or PBO plus SGE-treated (PBO + SGE) groups. The dietary concentration of SGE was selected as 1,000 ppm based on the result of the preliminary study in which rats were fed with a diet containing 2\% PBO and water containing SGE at $40 \mathrm{ppm}, 200 \mathrm{ppm}$ or 1,000 ppm for 4 weeks (unpublished observations). In the preliminary study, a dose-dependent increased expression of phase II drugmetabolizing enzyme genes was observed in real-time RT-PCR analyses. Therefore the dose of SGE was deter- mined to be 1,000 ppm. Four rats were housed in each stainless steel cage under the conventional conditions (12$\mathrm{hr}$ light/dark cycle; relative humidity, $55 \% \pm 5 \%$; temperature, $22 \pm 2{ }^{\circ} \mathrm{C}$, i.e., room temperature), and rats of the control and $\mathrm{PBO}$ groups or PBO $+\mathrm{SGE}$ group allowed ad libitum access for 2 weeks to tap water containing 0 or 1,000 ppm SGE. All rats were provided a commercial powdered-basal diet (MF, Oriental Yeast Industries Co., Ltd., Tokyo, Japan) for 2 weeks. In this experiment, we employed a two-stage rat liver carcinogenesis model in a medium-term rat liver bioassay (Fig. 1; Shirai, 1997). All animals were administered a single intraperitoneal injection of $\mathrm{N}$-diethylnitrosamine (200 $\mathrm{mg} / \mathrm{kg}$ body weight) dissolved in saline in order to initiate hepatocarcinogenesis. Two weeks after injection, the animals of the PBO and $\mathrm{PBO}+\mathrm{SGE}$ groups were administered a powdered diet containing $2 \%$ PBO for 7 weeks. This treatment period is generally 6 weeks, but the treatment period was changed to 7 weeks because of the man-power reason for the conduct of various examinations at the final necropsy. In addition, animals of the PBO + SGE group were subjected to concurrent administration of water containing 1,000 ppm SGE for 7 weeks. The PBO dose was determined based on our previous study, which was also conducted on a two-stage rat hepatocarcinogenesis model (Muguruma et al., 2007). In order to enhance hepatocellular proliferation, all rats were subjected to twothirds partial hepatectomy at 1 week after PBO feeding. Seven rats died postoperatively within 1 week and 29 rats survived ( 8 rats in the control group, 10 in the $\mathrm{PBO}$ group, and 11 rats the $\mathrm{PBO}+\mathrm{SGE}$ group). Body weight and food consumption were measured once a week. The experiment was performed in accordance with the guidelines for animal experimentation of the Faculty of Agriculture, Tokyo University of Agriculture and Technology. At the end of the treatment period, 3 animals from each group were used for the measurement of microsomal ROS production. Other animals were killed by exsanguination from the abdominal aorta under ether anesthesia and necropsied. Their livers were excised, weighed and either fixed in neutral buffered formalin or in Methacarn (methanol:chloroform:acetic acid $=6: 3: 1$ ) for histopathological examination or cut into small pieces, frozen in RNA later (QIAGEN, Hilden, Germany), and stored at $-80^{\circ} \mathrm{C}$ until analysis.

\section{Histopathology and morphometry}

The livers of all animals from the control, PBO and PBO + SGE groups were examined histopathologically. The livers that were fixed in Methacarn were embedded in paraffin wax, sectioned and stained with hematoxylin 
SGE exerts antioxidant activity by inducing phase II enzymes.

0 $2 \quad 4 \quad 5$ 11 weeks

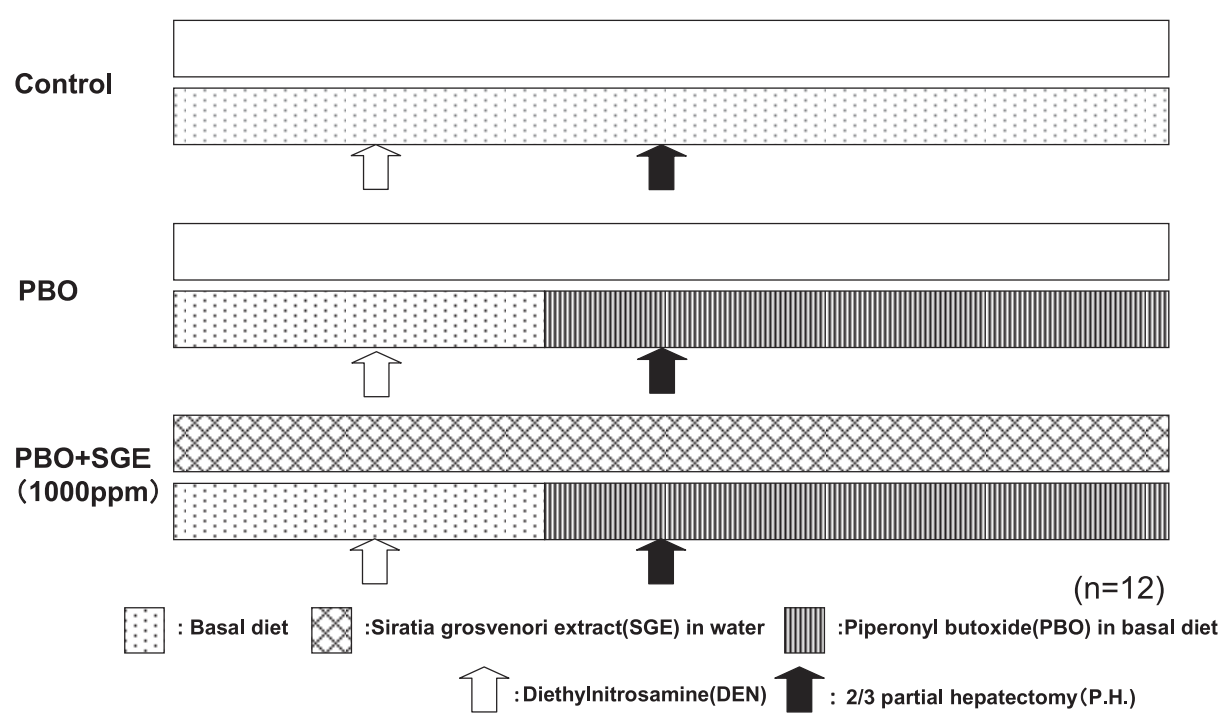

Fig. 1. Experimental design.

and eosin. Schmorl staining was additionally performed for the determination of lipofuscin deposition in the livers of the animals.

\section{Immunohistochemistry}

The sections fixed in Methacarn were used for immunohistochemical staining for glutathione $S$-transferase placental form (GST-P) and proliferating cell nuclear antigen (PCNA) by the avidin-biotin complex method. Immunostaining for GST-P was performed according to a previously described method (Muguruma et al., 2007). Since one rat of the control group had severe bile duct proliferation due to the surgical error of partial hepatectomy, this animal was excluded from the immunohistochemical evaluation. The GST-P-positive liver cell foci in each group (4 rats in the control group, 7 in the $\mathrm{PBO}$ group and 8 in the PBO + SGE group) were determined by quantitative analysis using a computer-assisted microscope (EX51; OLYMPUS Corp., Tokyo, Japan). The numbers and areas of the foci with cross-sectional areas $>0.01 \mathrm{~mm}^{2}$ and the total area of the liver sections were measured using a computer-assisted image analyzer (NIH Image, a free PC version of Image, called as Scion Image for Windows available from Scion Corporation ) to obtain the respective values in terms of per $\mathrm{cm}^{2}$ of the liver section.

For immunostaining for PCNA, deparaffinized liver sections were denatured by immersing in $2 \mathrm{~N} \mathrm{HCL}$ for
30 min in order to hydrolyze DNA. The sections were then rinsed in 2 changes of distilled water and phosphatebuffered saline ( $\mathrm{pH}$ 7.4). Immunostaining was performed using a mouse anti-PCNA antibody (Clone PC10; 1:300; DAKO, Glostrup, Denmark). The number of PCNA-positive cells among approximately 2,000 hepatocytes was then counted.

\section{Measurement of microsomal ROS production in the liver}

The liver microsomes were obtained from 3 rats of each group. ROS production was measured as described previously (Muguruma et al., 2006). To examine ROS generation in relation to metabolic activation by CYPs, the pooled sample from the PBO group was treated with SKF525A, a non-specific P450 inhibitor of mixed-function oxidases. The formation of ROS was detected as a fluorescent signal and was measured by a microplate reader (excitation, $485 \mathrm{~nm}$; emission, $528 \mathrm{~nm}$ ).

\section{Determination of TBARS content}

Lipid peroxidation in the livers was assessed by quantitation of the generated thiobarbituric acid-reactive substances (TBARS; Wasoxicz et al., 1993) in each group (4 rats in the control group, 6 in the PBO group and 6 in the $\mathrm{PBO}+\mathrm{SGE}$ group). Briefly, $100 \mu \mathrm{l}$ of the liver homogenate in $1.15 \% \mathrm{KCl}, 100 \mu \mathrm{l}$ of $8.1 \%$ sodium dodecyl sul- 
phate, $750 \mu \mathrm{l}$ of $20 \%$ acetic acid ( $\mathrm{pH} 3.5$ ), $25 \mu \mathrm{l}$ of $0.8 \%$ butylated hydroxytoluene, $750 \mu \mathrm{l}$ of $0.8 \%$ thiobarbituric acid and $350 \mu \mathrm{l}$ of distilled water were mixed in that order, heated at $95^{\circ} \mathrm{C}$ for $60 \mathrm{~min}$, and then cooled. The reaction mixture was centrifuged at $3,000 \mathrm{rpm}$ for $10 \mathrm{~min}$ after adding $0.5 \mathrm{ml}$ of distilled water and $2.5 \mathrm{ml} n$-butanol and pyridine $(15: 1 \mathrm{v} / \mathrm{v})$. The absorbance of the resulting solution was determined spectrophotometrically at 532 nm using the Synergy HT Multi-Detection Microplate Reader (BioTek, VT, USA).

\section{Determination of GST and GSH-Px enzyme activities}

The mixture used for measuring glutathione $S$-transferase (GST) activity contained $1 \mathrm{mM}$ glutathione, $1 \mathrm{mM}$ 1-chloro-2,4-dinitrobenzene (CDNB) in ethanol, 0.025 $\mathrm{ml}$ of cytosolic sample (containing $20 \mu \mathrm{g}$ of protein) and $0.1 \mathrm{M} \mathrm{Na}^{+} / \mathrm{Ka}^{+}$phosphate buffer ( $\mathrm{pH} 6.5$ ). The product obtained from the reaction between the thiol group of glutathione and the electrophilic group of CDNB was read spectrophotometrically at $340 \mathrm{~nm}\left(\varepsilon=9.6 \mathrm{mM}^{-1} \mathrm{~cm}^{-1}\right)$ (Habig et al., 1974). CDNB is a wide range of GST substrate, especially GST- $m 1$ and GST- $m 3$ (Jensson et al., 1986, Mannervik et al., 1985). The mixture used for measuring glutathione peroxidase (GSH-Px) activity contained $200 \mathrm{U} / \mathrm{ml}$ glutathione reductase, $20 \mathrm{mM}$ glutathione, $10 \mathrm{mM}$ EDTA, $10 \mathrm{mM} \mathrm{NaN}_{3}, 5 \mathrm{mM} \mathrm{NADPH}, 5$ $\mathrm{mM} \mathrm{H}_{2} \mathrm{O}_{2}$ and $0.1 \mathrm{M} \mathrm{Na}^{+} / \mathrm{Ka}^{+}$phosphate buffer ( $\mathrm{pH} 7.0$ ). The decrease in absorbance of NADPH was measured at $340 \mathrm{~nm}$ for $4 \mathrm{~min}\left(\varepsilon=6.22 \times 10^{3} \mathrm{M}^{-1} \mathrm{~cm}^{-1}\right)$ (Flohe et al., 1984).

\section{DNA microarray analysis}

As a screening analysis to examine fluctuations of variable genes attributable to coadministration of $\mathrm{PBO}$ and SGE, the livers from 1 PBO-treated and 1 PBO + SGEtreated rat were each analyzed by Oligo GEArray Rat Toxicology and Drug Resistance Microarray (ORN-401; SuperArray Bioscience Corp., Frederick, MD). Total RNA was extracted using TRIzol (Invitrogen Corp., Carlsbad, CA) according to the manufacturer's instructions. By using the TrueLabeling-AMP kit (SuperArray Bioscience Corp.), cRNA was synthesized from $3 \mu \mathrm{g}$ of total RNA by conversion of the total RNA to a biotin-labeled cRNA probe through the cDNA synthesis step. After purification of the biotin-labeled cRNA probes by using the ArrayGrade ${ }^{\mathrm{TM}}$ cRNA Clean-up Kit (SuperArray Bioscience Corp.), the array membranes were hybridized overnight with the biotin-labeled probes at $60^{\circ} \mathrm{C}$. The membranes were then washed twice with $2 \times$ saline-sodium citrate buffer (SSC)/1\% sodium dodecyl sulfate (SDS) followed by washing twice with $0.1 \times \mathrm{SSC} / 1 \% \mathrm{SDS}$ at $60^{\circ} \mathrm{C}$ for $15 \mathrm{~min}$ each. Chemiluminescent detection steps were performed by subsequent incubation of the membranes with alkaline phosphatase-conjugated streptavidin and CDP-Star substrate followed by exposure to Hyperfilm ${ }^{\mathrm{TM}}$ ECL X-ray film (Amersham Biosciences UK Ltd., Buckinghamshire, UK). The image data obtained from GEArray were analyzed using the GEArray Expression Analysis Suite software (http://www.geasuite.superarray. com/index.jsp ) after correcting for background noise by subtraction of the minimum value and normalizing to the value of 2 housekeeping genes (Ppia and GAPDH). For each spot, the ratio of intensities between the PBO and $\mathrm{PBO}+\mathrm{SGE}$ groups was analyzed. Gene expressions in the $\mathrm{PBO}+\mathrm{SGE}$ group were considered to be significant if their fold change values were $>1.5$-fold, as compared to the PBO group.

\section{Real-time RT-PCR}

Real-time RT-PCR analysis was performed using liver tissue from each group (4 animals in the control group, 5 in the PBO group and 5 in the PBO + SGE group). Since one rat of the control group had severe bile duct proliferation due to the surgical error of partial hepatectomy, this animal was excluded from the real-time RT-PCR. Based on the results of the present cDNA microarray analyses and our previous study on PBO-induced hepatocarcinogenesis (Muguruma et al., 2007), genes related to oxidative stress were selected for quantitative real-time RT-PCR analyses. cDNA was synthesized from $2 \mu \mathrm{g}$ of RNA in the presence of dithiothreitol (DTT), dNTPs, random primers, RNaseOUT and SuperScript ${ }^{\mathrm{TM}}$ III Reverse Transcriptase (Invitrogen Corp.) in $20 \mu \mathrm{l}$ total reaction mixture. Realtime RT-PCR was performed using the SYBR ${ }^{\circledR}$ Green PCR Master Mix (Applied Biosystems, Foster City, CA, USA) and the ABI PRISM 7000 Sequence Detection System (Applied Biosystems) to validate the microarray results, according to the SYBR ${ }^{\circledR}$ Green PCR Master Mix protocol. The PCR primers shown in Table 1 were designed using the Primer Express software (Applied Biosystems). The amount of transcripts of the target genes normalized to an endogenous reference gene, $M y o 1 b$, and relative to a control was determined by the ${ }^{2 \triangle} \mathrm{CT}$ method (Livak and Schmittgen, 2001).

\section{Statistical analysis}

Statistical analyses were performed using a statistical software (StatLight; Yukms Co., Ltd., Japan), and all results have been presented as the mean \pm SD. The significance level of the difference between the control and the $\mathrm{PBO}$ or $\mathrm{PBO}+\mathrm{SGE}$ groups, and that between the PBO 
SGE exerts antioxidant activity by inducing phase II enzymes.

Table 1. Sequence of primers used for real-time RT-PCR analysis.

\begin{tabular}{|c|c|c|c|}
\hline Accession no. & Symbol & Forward primer & Reverse primer \\
\hline NM_080681 & Abcc3 & TCCCACTTCTCGGAGACAGTAAC & CTTAGCATCACTGAGGACCTTGAA \\
\hline NM_013215 & Afar & CCGCTTCTTTGGGAATCCAT & GGCGATGCCATTGAAGTGT \\
\hline AA800587 & Gpx2 & AC CG ATC CC AAG CTC ATC ATC AT & TCTCAAAGTTCCAGGACACATCTG \\
\hline X78847 & Gst-yc2 & AAGCTGAGCAGGGCTGATGT & ACAATGCCTGGGTCCATCTC \\
\hline NM_017013 & Gst-a2 & CAACTACATCGCCACCAAATATG & GCCTTCGGTGTACATGTCAATC \\
\hline NM_017014 & Gst-m1 & GAACGTTCGCGGACTTACTCA & ACGTATCTCTTCTCCTCATAGTTTGAATCT \\
\hline NM_031154 & Gst-m3 & GCCATCCTGCGCTATCTTG & CACGAATCCTCTCCTCTTCTGTCT \\
\hline M13506 & UDPGTR-2 & CTGAAGCAGAGCCCTGAGAGA & GGGAAGGCACTGGCATGA \\
\hline NM_057105 & Ugtla6 & TGGCTACCCCAAAACGATCT & ATACCATGGGAACCGGAGTGT \\
\hline NM_017000 & Nqo1 & TCCGCCCCCAACTTCTG & TCTGCGTGGGCCAATACA \\
\hline NM_053906 & Gsr & GCCCACGGTTCCTCATGA & GAAAGAACCCATCACTGGTTATCC \\
\hline NM_017353 & Slc7a5 & CCTACGGAGGATGGAACTATCTGA & TGGGCAAGGAGATGATGATG \\
\hline NM_012541 & Cypla1 & \multicolumn{2}{|c|}{ This gene primer set was obtained from Superarray Bioscience } \\
\hline NM_031762 & Cdkn1b & TTCCGCCTGCAGAAACCTCTT & TCTCGGCAGTGCTTCTCCAAGT \\
\hline NM_024127 & Gadd45a & CACCATAACTGTCGGCGTGTA & GGCACAGGACCACGTTGTC \\
\hline NM_022246 & $\operatorname{Rad} 50$ & TGGCCCCTGGCAGTGA & AACTTCGCACGCCCAGAGT \\
\hline NM_012600 & ME1 & CGACCAGCAAAGCTGAGTGTT & CTGCCGCTGGCAAAGATC \\
\hline NM_013149 & AhR & CATCCTGGAAATTCGAACCAA & GCATCACAGCCAATAGGTGTGA \\
\hline NM_053986 & Myo1b & GCAGGAGAAAGTTTCAACCACAT & AACCGGCTGTAGAGGTTTTTAGC \\
\hline
\end{tabular}

and PBO + SGE groups were determined by Student's $t$ test when the intergroup variance was proved to be homogeneous using the test for equal variance. If a significant difference was observed in the variance, Welch's $t$-test was performed. A $P$ value of less than 0.05 was considered to be statistically significant.

\section{RESULTS}

\section{Body and liver weights}

The body weight gain in the PBO-treated rats was significantly inhibited compared with that in the control group, as shown in our earlier study (data not shown; Muguruma et al., 2007). As compared to the control group, the final body weight was significantly decreased in both PBO and PBO + SGE groups. However, the values did not differ between the PBO-treated groups (Table 2 ). Both absolute and relative liver weights were signifi- cantly increased in both the PBO-treated groups as compared to the control group (Table 2). However, none of the values differed between the PBO and PBO + SGE groups.

\section{Histopathology}

Centrilobular hepatocytic hypertrophy was evident on microscopic examination of the livers of the PBO and $\mathrm{PBO}+\mathrm{SGE}$ groups (data not shown). In these groups, the hepatocytes were enlarged due to an increase in the cytoplasmic area. Schmorl staining revealed an increase in the deposition of lipofuscin, one of the markers of oxidative stress response, in the $\mathrm{PBO}$ and $\mathrm{PBO}+\mathrm{SGE}$ groups (Fig. $2 \mathrm{a}-\mathrm{c})$. However, there was no marked difference in the severity between these two groups.

\section{Immunohistochemical analysis}

The data for immunohistochemical analysis of the 
H. Yasuno et al.

Table 2. Body and liver weights of rats given PBO and/or SGE after DEN initiation.

\begin{tabular}{|c|c|c|c|c|}
\hline & \multirow[t]{2}{*}{ No. of rats examined } & \multirow[t]{2}{*}{ Body weight (g) } & \multicolumn{2}{|l|}{ Liver weight } \\
\hline & & & Absolute (g) & Relative (\%) \\
\hline Control & 4 & $273.63 \pm 16.8$ & $6.86 \pm 0.68$ & $2.5 \pm 0.21$ \\
\hline PBO & 7 & $209.82 \pm 12.8^{* *}$ & $11.74 \pm 0.77 * *$ & $5.6 \pm 0.12 * *$ \\
\hline $\mathrm{PBO}+\mathrm{SGE}$ & 8 & $205.55 \pm 8.69^{* *}$ & $11.66 \pm 0.64 * *$ & $5.67 \pm 0.13 * *$ \\
\hline
\end{tabular}

Results are mean \pm S.D. for indicated number of animals for each group.

** $P<0.01$ vs. Control.

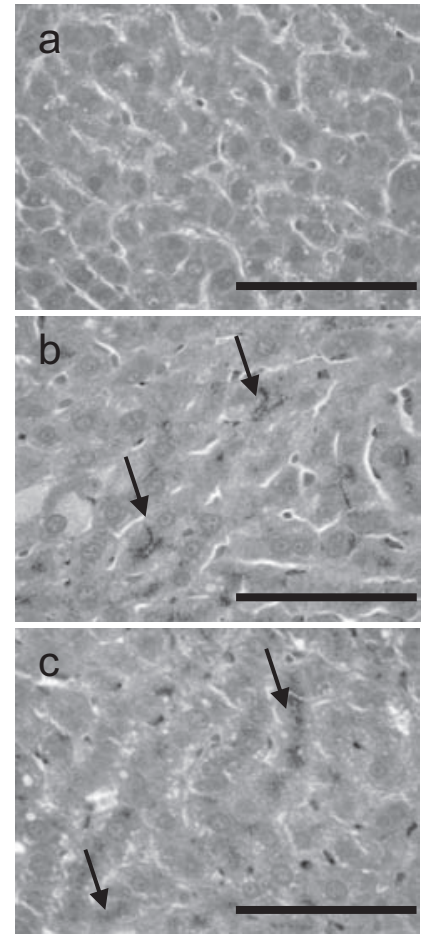

Fig. 2. Representative light microscopic views of Schrmol staining of the liver in each group (a: control, b: PBO, $\mathrm{c}$ : $\mathrm{PBO}+\mathrm{SGE}$ ). Lipofuscin deposition (arrows) in the liver of the animals of the $\mathrm{PBO}$ and $\mathrm{PBO}+\mathrm{SGE}$ groups is more prominent as compared to the control group. Bar $=200 \mu \mathrm{m}$.

GST-P-positive liver cell foci and PCNA-positive cells are shown in Table 3. The number and area of GST-Ppositive foci were significantly increased in both $\mathrm{PBO}$ and $\mathrm{PBO}+\mathrm{SGE}$ groups as compared to the control group. When the values of both PBO-treated groups were compared, a significant increase was observed in the area but not in the number of the GST-P-positive foci in the PBO
+ SGE group. The PCNA-positive cell ratio was significantly increased in both the PBO-treated groups as compared to that in the control group; however, the values did not differ between the PBO and PBO + SGE groups.

\section{Generation of ROS}

Measurement of ROS production in the cell-free system of isolated liver microsomes obtained from the PBO and $\mathrm{PBO}+\mathrm{SGE}$ groups revealed a significant increase in ROS production as compared to the control group (Fig. 3 ). However, ROS production did not differ significantly between the PBO and PBO + SGE groups. Addition of SKF525A to the pooled sample from the PBO group showed inhibition of ROS production.

\section{Measurement of TBARS}

Hepatic TBARS levels, measured using malondialdehyde-a metabolite of oxidized lipids-as a standard, reflect the extent of lipid peroxidation. TBARS values were significantly increased in both $\mathrm{PBO}$ and $\mathrm{PBO}+\mathrm{SGE}$ groups as compared to that in the control group (Table 4). However, the TBARS value in the PBO + SGE group was significantly lower than that in the $\mathrm{PBO}$ group.

\section{GST and GSH-Px activity}

Hepatic GST activity was significantly increased in the PBO and PBO + SGE groups (Table 4), and was significantly higher in the latter as compared to the former. Hepatic GSH-Px activity in the PBO and control groups was similar. However, that in the PBO + SGE group was significantly higher than the control and $\mathrm{PBO}$ groups (Table 4).

\section{cDNA microarray and real-time RT-PCR analysis}

As compared to the PBO group, in the PBO + SGE group, microarray analysis of the liver showed a $>1.5$-fold increase in the mRNA levels of cyclin-dependent kinase inhibitor 1B $(C d k n 1 b)$, growth arrest and DNA-damage- 
SGE exerts antioxidant activity by inducing phase II enzymes.

Table 3. Quantitative data for GST-P-positive foci and PCNA-positive cells in livers of rats given PBO and/or SGE after DEN-initiation.

\begin{tabular}{|c|c|c|c|c|}
\hline & \multirow[t]{2}{*}{ No. of rats examined } & \multicolumn{2}{|c|}{ GST-P-positive foci $\left(>0.01 \mathrm{~mm}^{2}\right)$} & \multirow{2}{*}{$\begin{array}{l}\text { PCNA-positive cells } \\
\text { Number (No./2000 cells) }\end{array}$} \\
\hline & & Numbers $\left(\right.$ No. $\left./ \mathrm{cm}^{2}\right)$ & Areas $\left(\mathrm{mm}^{2} / \mathrm{cm}^{2}\right)$ & \\
\hline Control & 4 & $29.99 \pm 4.87$ & $0.57 \pm 0.13$ & $51.6+6.5$ \\
\hline $\mathrm{PBO}$ & 7 & $70.48 \pm 23.49 * *$ & $1.59 \pm 0.39 * *$ & $112.3+34 *$ \\
\hline $\mathrm{PBO}+\mathrm{SGE}$ & 8 & $79.6 \pm 17.97 * *$ & $2.75 \pm 1.22 * *$ & $107.8+30.9^{*}$ \\
\hline
\end{tabular}

Results are mean \pm S.D. for indicated number of animals for each group.

*,** $P<0.05$ or $P<0.01$ vs. Control, respectively.

\# $P<0.05$ vs. $\mathrm{PBO}$
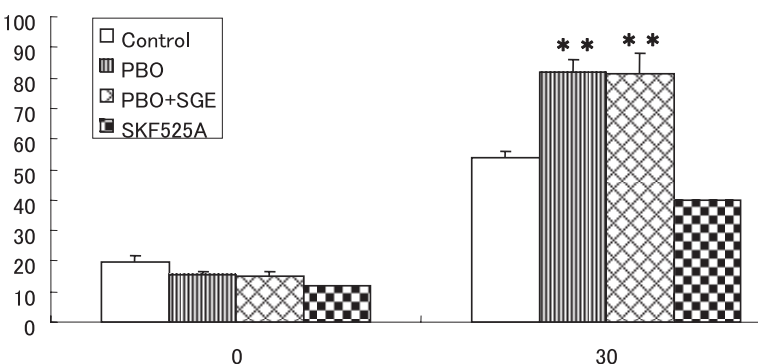

Fig. 3. Effects of PBO on hepatic microsomal ROS production. The column represents the mean $\pm \mathrm{SD}$. Hepatic microsomes isolated from the control, $\mathrm{PBO}$ and $\mathrm{PBO}+$ SGE groups were measured in a cell-free system $(n=3$ in each group). The pooled sample from the PBO group was used for examination of the effect of SKF525A, a nonspecific $\mathrm{P} 450$ inhibitor of mixed-function oxidases. $* * P<0.01$ vs. the control group.

inducible 45 alpha (Gadd45a), rat homolog of Rad50 (Rad50), epoxide hydrolase 1, microsomal, (Ephxl), $\mathrm{NAD}(\mathrm{P}) \mathrm{H}$ dehydrogenase quinone 1 (Nqol), glutathione reductase $(G s r)$, glutathione peroxidase 2 (Gpx2), and the GST isoforms pi2, mu3, and theta 1 (Gstp2, Gstm3, and Gstt1) (Table 5). To validate the mRNA expression data obtained from the microarray analysis, real-time RTPCR analysis was performed. In addition, several xenobiotic-metabolizing genes associated with oxidative stress responses and the genes that were found to be up-regulated in our previous study on PBO (Muguruma et al., 2007) were chosen, and consequently, 16 genes in all were selected for real-time RT-PCR analysis. Gene expression levels obtained by real-time RT-PCR were mostly parallel to those obtained by microarray analysis. Results of these analyses are summarized in Table 6. As reported in our previous study, mRNA levels of the phase II enzyme genes, such as Nqol, Gsr, UDP-glucuronosyltransferase phenobarbital-inducible form (Udpgtr-2), aflatoxin B1 aldehyde reductase (Afar) and Gpx2, were significantly increased in the livers of the rats from both the PBOtreated groups, as compared to the control group. These genes were further up-regulated and their mRNA levels, significantly increased in the PBO + SGE group, as compared to the PBO group. Similarly, the mRNA expression of ATP-binding-related genes, such as $A b c c 3$ and oxidoreductase activity-related genes (Me1), which were significantly up-regulated in the $\mathrm{PBO}$ group were further increased in the PBO + SGE group. On the other hand, a phase I enzyme gene, i.e., Cypla1, showed significant upregulation in the $\mathrm{PBO}$ and $\mathrm{PBO}+\mathrm{SGE}$ groups compared to the control group, as in our previous study (Muguruma et al., 2007). However, no significant difference was observed in its expression between the $\mathrm{PBO}$ and $\mathrm{PBO}+$ SGE groups. In addition, the transcription level of $A h R$, which is related to the induction of Cyplal expression, was also significantly increased in the PBO and PBO + SGE groups, as compared to the control group. The mRNA levels of Nrf 2-dependent genes, such as Gst genes (Gsta2, Gstm1, Gstm3, and Gstyc2), were also increased in the PBO group, as compared to the control group. Among them, Gstm1 and Gstyc2 were further and significantly up-regulated in the PBO + SGE group, as compared to the PBO group. Furthermore, cell cycle arrest and DNA repair-related genes, such as Cdkn1b, Gadd45a, and Rad50 were significantly up-regulated by coadministration of SGE with PBO.

\section{DISCUSSION}

Chemical carcinogens that act by generating free radical metabolites are associated with many biochemical and molecular changes that induce oxidative stress leading to tumor promotion. In accordance with our previous 
Table 4. Enzyme activities in livers of rats given PBO and/or SGE after DEN-initiation.

\begin{tabular}{lllll}
\hline No. of rats examined & $\begin{array}{l}\text { TBARS levels } \\
\text { (nmol MDA/g liver) }\end{array}$ & $\begin{array}{l}\text { GST activity } \\
\text { (nmol CDNB conjugate } \\
\text { formed/min/mg protein) }\end{array}$ & $\begin{array}{l}\text { GSH-px activity } \\
\text { (nmol NADPH } \\
\text { oxidized/min/mg protein) }\end{array}$ \\
\hline Control & 4 & $154.84 \pm 21.18$ & $77.2 \pm 13.7$ & $8.42 \pm 0.59$ \\
PBO & 6 & $228.62 \pm 19.4^{* *}$ & $141.39 \pm 4.22^{* *}$ & $9.52 \pm 1.37$ \\
PBO+SGE & 6 & $195.16 \pm 11.31^{*}, \#$ & $154.7 \pm 12.69^{* *}, \#$ & $12.63 \pm 2.36^{* *, \#}$ \\
\hline
\end{tabular}

Results are mean \pm S.D. for indicated number of animals for each group.

*** $P<0.05$ or $P<0.01$ vs. Control, respectively

${ }^{*} \mathrm{PBO}$ at $P<0.05$ vs. PBO

Table 5. Microarray data: Up-regulated ( $>1.5$ fold) genes in the liver of a PBO + SGE-treated rat compared to a PBO-treated rat.

\begin{tabular}{|c|c|c|c|c|}
\hline Function & Symbol & Description & Ratio (PBO+SGE/PBO) & Accession no. \\
\hline \multicolumn{5}{|c|}{ Drug metabolizing enzymes } \\
\hline & $G s r$ & Glutathione reductase & 6.85 & NM_053906 \\
\hline & Gpx2 & Glutathione peroxidase 2 & 4.86 & NM_183403 \\
\hline & Nqo1 & $\mathrm{NAD}(\mathrm{P}) \mathrm{H}$ dehydrogenase, quinone 1 & 3.55 & NM_017000 \\
\hline & Gstp2 & Glutathione S-transferase, pi 2 & 5.09 & NM_138974 \\
\hline & Gstm3 & Glutathione S-transferase, mu type 3 & 2.83 & NM_031154 \\
\hline & Gstt1 & Glutathione S-transferase theta 1 & 2.72 & NM_053293 \\
\hline & Ephxl & Epoxide hydrolase 1, microsomal & 4.77 & NM_012844 \\
\hline \multicolumn{5}{|c|}{ DNA repair } \\
\hline & Rad50 & RAD50 homolog (S. cerevisiae) & 2.54 & NM_022246 \\
\hline \multicolumn{5}{|c|}{ Cell cycle arrest } \\
\hline & Gadd45a & Growth arrest and DNA-damage-inducible 45 alpha & 5.81 & NM_024127 \\
\hline & $C d k n 1 b$ & Cyclin-dependent kinase inhibitor 1B & 3.88 & NM_031762 \\
\hline \multicolumn{5}{|l|}{ Apoptosis } \\
\hline & $\operatorname{Prd} x 2$ & Peroxiredoxin 2 & 1.5 & NM_017169 \\
\hline & Ddit3 & DNA-damage inducible transcript 3 & 4.21 & NM_024134 \\
\hline
\end{tabular}

studies on mice and rats (Muguruma et al., 2006, 2007), we successfully reproduced the tumor-promoting activity of PBO in response to oxidative stress. We also confirmed the role of lipid peroxidation in the tumor-promoting activity. A striking finding of the present study was that SGE coadministration inhibited lipid peroxidation. Such a decrease in lipid peroxidation by SGE was also reported in type 2 diabetic Goto-Kakizaki rats (Suzuki et al., 2007). Antioxidants exert their action against oxidant-mediated damage by impairment of lipid peroxidation or due to their antioxidant activity (Shahjahan et al., 2005). On the other hand, in the cell-free microsomal system in the present study, there was no marked difference in the ROS production between the PBO and PBO + SGE 
SGE exerts antioxidant activity by inducing phase II enzymes.

Table 6. Real-time RT-PCR analysis of mRNA expression in liver tissues of the control, PBO and PBO + SGE groups.

\begin{tabular}{|c|c|c|c|}
\hline Symbol & Control $(n=4)$ & $\mathrm{PBO}(\mathrm{n}=5)$ & $\mathrm{PBO}+\mathrm{SGE}(\mathrm{n}=5)$ \\
\hline Gpx2 & $1.29 \pm 0.9$ & $14.07 \pm 2.03^{* *}$ & $27.88 \pm 4.48^{* *}$ \\
\hline Nqol & $1.09 \pm 0.36$ & $2.62 \pm 0.7^{* *}$ & $4.07 \pm 0.43 * *$, \# \\
\hline Gsr & $1 \pm 0.11$ & $2.85 \pm 0.45^{* *}$ & $4.21 \pm 0.52 * *$ \\
\hline Afar & $1 \pm 0.12$ & $5.06 \pm 1.7^{*}$ & $9.12 \pm 1.72 * *$ \\
\hline$G S T-y c 2$ & $1.34 \pm 1.24$ & $27.88 \pm 10.91 * *$ & $46.73 \pm 11.07 * *$ \\
\hline GST-m1 & $1 \pm 0.09$ & $22.62 \pm 4.4^{* *}$ & $33.11 \pm 7.64^{* *}$ \\
\hline$G S T-m 3$ & $1.02 \pm 0.2$ & $1.73 \pm 0.22 * *$ & $2 \pm 0.21 * *$ \\
\hline GST-a2 & $1.01 \pm 0.18$ & $1.91 \pm 0.35^{* *}$ & $2.44 \pm 0.42 * *$ \\
\hline Udpgtr2 & $1.01 \pm 0.12$ & $9.76 \pm 2.34^{* *}$ & $14.89 \pm 1.53 * *$ \\
\hline$A b c c 3$ & $1.181 \pm 0.74$ & $22.19 \pm 3.47^{* *}$ & $37.6 \pm 7.91^{* *}$ \\
\hline $\mathrm{Mel}$ & $1.01 \pm 0.14$ & $3.15 \pm 0.81^{* *}$ & $4.22 \pm 0.42 * *$ \\
\hline Gadd45a & $1.05 \pm 0.4$ & $2.16 \pm 0.66^{*}$ & $3.75 \pm 0.9^{* *}$ \\
\hline$C d k l 1 b$ & $1.02 \pm 0.25$ & $1.76 \pm 0.67$ & $3.72 \pm 1.04^{*}, \#$ \\
\hline Rad50 & $1.15 \pm 0.57$ & $1.62 \pm 0.43$ & $3.06 \pm 0.76^{* *}$ \\
\hline Cyplal & $1.59 \pm 1.43$ & $30.74 \pm 12.5^{* *}$ & $35.79 \pm 14.04^{* *}$ \\
\hline$A h R$ & $1.02 \pm 0.22$ & $1.93 \pm 0.46^{* *}$ & $1.87 \pm 0.35^{* *}$ \\
\hline
\end{tabular}

Results are mean \pm S.D. for indicated number of animals for each group.

*,** $P<0.05$ or $P<0.01$ vs. Control, respectively.

\#,\# $P<0.05$ or $P<0.01$ vs. PBO, respectively.

groups, suggesting that SGE was not associated with ROS generation during PBO metabolism through the induction of CYP1A1.

In the present study, mRNA levels of phase II drugmetabolizing enzyme genes, such as Gpx2, Nqo1, Gsr, Udpgtr2, Afar, Gstm1, and Gstyc2, were increased by coadministration of SGE with PBO. GST (GST- $m 1$ and GST- $m 3$ ) and GSH-Px activities also increased in parallel to the changes in mRNA expression. Gpx2, Nqo1, Gsr, Afar, Gstm 1 and Gstyc2 contain an antioxidant response element (ARE), i.e., a consensus sequence motif for the transcription factor Nrf 2, in their promoter regions. Also, the rat Udpgtr2 gene is homologous to the mouse Ugt2b1 gene that is also known to be regulated by Nrf 2 (Shelby and Klaassen, 2006). In addition, Me1, a NADPH-generating malic enzyme gene that is known to be flanked by ARE at the promoter region (Li et al., 2002), was shown to be up-regulated by SGE coadministration in the present study. Abcc3 is an ATP-binding cassette gene coding for a phase III enzyme that is known to function in the transport of organic anion-conjugating chemicals and play a role in hepatic detoxification and tissue-specific distribution; it was also shown to have been up-regulated by SGE coadministration. $A b c c 3$ has been shown to be induced by activators of the constitutive androstane receptor and an ARE (Cherrington et al., 2002). Thus, the genes up-regulated by SGE administration that were examined here were all regulated directly by Nrf 2-mediated transcription or indirectly following transcriptional activation of Nrf 2-dependent genes. Many antioxidants exert their chemopreventive activity by inducing phase II enzymes (Kim et al., 2006; Wu et al., 2006; Lee et al., 2007; Yates et al., 2006). Therefore, the results of the present study suggest that SGE also exerts antioxidant effects through activation of an ARE.

Liver weights and the number of GST-P-positive foci remained unchanged by coadministration of SGE; however, SGE coadministration increased the area of the GST- 
P-positive foci, suggesting an increase in the number of GST-P-positive cells per focus. On the other hand, the number of PCNA-positive cells was unaltered by SGE coadministration. If SGE has a tumor-promoting action in the liver, the number of GST-P positive foci and PCNA positive cells should also be increased. In this respect, it may be concluded that the action of SGE on the liver may not be related to its tumor-promoting activity.

In conclusion, the results of the present study suggest that SGE exerts antioxidant activity by inducing phase II enzymes through the Nrf 2-Keap1-ARE transcriptional machinery, although the antioxidant activity of SGE is neither effective nor sufficient for suppression of PBOpromoted early hepatocarcinogenic responses. However, it is noteworthy that several antioxidant genes were fluctuated by SGE treatment in the ROS-mediated hepatocarcinogenesis, and these findings are very important for the future clarification of mechanism of SGE antioxidant activity. Further studies are necessary to determine the conditions under which SGE effectively shows cancer-preventive activity.

\section{REFERENCES}

Bansal, A.K., Bansal, M., Soni, G. and Bhatnagar, D. (2005): Protective role of Vitamin $\mathrm{E}$ pre-treatment on N-nitrosodiethylamine induced oxidative stress in rat liver. Chem. Biol. Interact., 156, 101-111.

Beamand, J.A., Price, R.J., Phillips, J.C., Butler, W.H., Jones, G.D., Osimitz, T.G., Gabriel, K.L., Preiss, F.J. and Lake, B.G. (1996) Lack of effect of piperonyl butoxide on unscheduled DNA synthesis in precision-cut human liver slices. Mutat. Res., 371, 273282.

Butler, W.H., Gabriel, K.L., Osimitz, T.G. and Preiss, F.J. (1996): Lack of genotoxicity of piperonyl butoxide. Mutat. Res., 371, 249-258.

Cherrington, N.J., Hartley, D.P., Li, N., Johnson, D.R. and Klaassen, C.D. (2002): Organ distribution of multidrug resistance proteins 1, 2, and 3 (Mrp1, 2, and 3) mRNA and hepatic induction of Mrp3 by constitutive androstane receptor activators in rats. J. Pharmacol. Exp. Ther., 300, 97-104.

Flohe, L. and Gunzler, W.A. (1984): Assays of glutathione peroxidase. Methods. Enzymol., 105, 114-121.

Habig, W.H., Pabst, M.J. and Jakoby, W.B. (1974): Glutathione STransferases. The first enzymatic step in mercapturic acid formation. J. Biol. Chem., 249, 7130-7139.

Hossen, M., Shinmei, Y., Jiang, S., Takubo, M., Tsumuro, T., Murata, Y., Sugiura, M. and Kamei, C. (2005): Effect of Lo Han Kuo (Siraitia grosvenori Swingle) on Nasal Rubbing and Scratching Behavior in ICR Mice. Biol. Pharm. Bull., 28, 238241.

Jensson, H., Guthenberg, C., Ålin, P. and Mannervik, B. (1986): Rat glutathione transferase 8-8, an enzyme efficiently detoxifying 4hydroxyalk-2-enals. FEBS Lett., 203, 207-209.

Khan, N., Sharma, S., Alam, A., Saleem, M. and Sultana, S. (2001): Tephrosia purpurea ameliorates N-diethylnitrosamine and potassium bromate-mediated renal oxidative stress and toxicity in
Wistar rats. Pharmacol. Toxicol., 88, 294-299.

Kim, M.R., Lee, J.Y., Lee, H.H., Aryal, D.K., Kim, Y.G., Kim, S.K., Woo, E.R. and Kang, K.W. (2006): Antioxidative effects of quercetin-glycosides isolated from the flower buds of Tussilago farfara L. Food. Chem. Toxicol., 44, 1299-1307.

Lee, S.B., Cha, K.H., Selenge, D., Solongo, A. and Nho, C.W. (2007): The chemopreventive effect of taxifolin is exerted through ARE-dependent gene regulation. Biol. Pharm. Bull., 30, 1074-1079.

Li, J., Lee, J.M. and Johnson, J.A. (2002): Microarray analysis reveals an antioxidant responsive element-driven gene set involved in conferring protection from an oxidative stressinduced apoptosis in IMR-32 cells. J. Biol. Chem., 277, 388394.

Livak, K.J. and Schmittgen, T.D. (2001): Analysis of relative gene expression data using real-time quantitative PCR and the $2^{-\Delta \Delta \mathrm{C} T}$ Method. Methods. 25, 402-408.

Mannervik, B., Alin, P., Guthenberg, C., Jensson, H., Tahir, M.K., Warholm, M. and Jornvall, H. (1985): Identification of three classes of cytosolic glutathione transferase common to several mammalian species: correlation between structural data and enzymatic properties. Proc. Natl. Acad. Sci. USA, 82, 72027206.

Muguruma, M., Nishimura, J., Jin, M., Kashida, Y., Moto, M., Takahashi, M., Yokouchi, Y. and Mitsumori, K. (2006): Molecular pathological analysis for determining the possible mechanism of piperonyl butoxide-induced hepatocarcinogenesis in mice. Toxicology, 228, 178-187.

Muguruma, M., Unami, A., Kanki, M., Kuroiwa, Y., Nishimura, J., Dewa, Y., Umemura, T., Oishi, Y. and Mitsumori, K. (2007): Possible involvement of oxidative stress in piperonyl butoxide induced hepatocarcinogenesis in rats. Toxicology, 236, 61-75.

Okamiya, H., Mitsumori, K., Onodera, H., Ito, S., Imazawa, T., Yasuhara, K. and Takahashi, M. (1998) : Mechanistic study on liver tumor promoting effects of piperonyl butoxide in rats. Arch. Toxicol., 72, 744-750.

Roebuck, B.D., Curphey, T.J., Li, Y., Baumgartner, K.J., Bodreddigari, S., Yan, J., Gange, S.J., Kensler, T.W. and Sutter, T.R. (2003): Evaluation of the cancer chemopreventive potency of dithiolethione analogs of oltipraz. Carcinogenesis, 24, 19191928.

Shahjahan, M., Vani, G. and Shyamaladevi, C.S. (2005): Effect of Solanum trilobatum on the antioxidant status during diethyl nitrosamine induced and phenobarbital promoted hepatocarcinogenesis in rat. Chem. Biol. Interact., 156, 113-123.

Shelby, M.K. and Klaassen, C.D. (2006): Induction of rat UDPglucuronosyltransferases in liver and duodenum by microsomal enzyme inducers that activate various transcriptional pathways. Drug Metab. Dispos., 34, 1772-1778.

Shi, H., Hiramatsu, M., Komatsu, M. and Kayama, T. (1996): Antioxidant property of Fructus Momordicae extract. Biochem. Mol. Biol. Int., 40, 1111-1121.

Shirai, T. (1997): A medium-term rat liver bioassay as a rapid in vivo test for carcinogenic potential: a historical review of model development and summary of results from 291 tests. Toxicol. Pathol., 25, 453-460.

Song, F., Qi, X., Chen, W., Jia, W., Yao, P., Nussler, A.K., Sun, X. and Liu, L. (2007): Effect of Momordica grosvenori on oxidative stress pathways in renal mitochondria of normal and alloxaninduced diabetic mice Involvement of heme oxygenase-1. Eur. J. Nutr., 46, 61-69.

Suzuki, Y.A., Tomoda, M., Murata, Y., Inui, H., Sugiura, 
SGE exerts antioxidant activity by inducing phase II enzymes.

M. and Nakano, Y. (2007): Antidiabetic effect of long-term supplementation with Siraitia grosvenori on the spontaneously diabetic Goto-Kakizaki rat. Br. J. Nutr., 97, 770-775.

Takahashi, O., Oishi, S., Fujitani, T., Tanaka, T. and Yoneyama, M. (1994): Chronic toxicity studies of piperonyl butoxide in F344 rats: induction of hepatocellular carcinoma. Fundam. Appl. Toxicol., 22, 293-303.

Takasaki, M., Konoshima, T., Murata, Y., Sugiura, M., Nishino, H., Tokuda, H., Matsumoto, K., Ksai, R. and Yamazaki, K. (2003): Anticarcinogenic activity of natural sweeteners, cucurbitane glycosides, from Momordica grosvenori. Cancer Lett., 198, 37-42.

Takeo, E., Yoshida, H., Tada, N., Shingu, T., Matsuura, H., Murata, Y., Yoshikawa, S., Ishikawa, T., Nakamra, H., Ohsuzu, F. and Kohda, H. (2002): Sweet elements of Siraitia grosvenori inhibit oxidative modification of low-density lipoprotein. J. Atheroscler. Thromb., 9, 114-120.

Wasowicz, W., Nève J. and Peretz, A. (1993): Optimized steps in fluorometric determination of thiobarbituric acid-reactive substances in serum: importance of extraction $\mathrm{pH}$ and influence of sample preservation and storage. Clin. Chem., 39, 2522-2526.
Wu, C.C., Hsu, M.C., Hsieh, C.W., Lin, J.B., Lai, P.H. and Wung, B.S. (2006): Upregulation of heme oxygenase-1 by Epigallocatechin-3-gallate via the phosphatidylinositol 3-kinase/Akt and ERK pathways. Life. Sci., 78, 2889-2897.

Yates, M.S., Kwak, M.K., Egner, P.A., Groopman, J.D., Bodreddigari, S., Sutter, T.R., Baumgartner, K.J., Roebuck, B.D., Liby, K.T. and Yore, M.M., Honda T, Gribble GW, Sporn, M.B. and Kensler, T.W. (2006): Potent protection against aflatoxin-induced tumorigenesis through induction of $\mathrm{Nrf}$ 2-regulated pathways by the triterpenoid 1-[2-cyano-3-,12-dioxooleana1,9(11)-dien-28-oyl]imidazole. Cancer Res., 66, 2488-2494.

Yeh, C.T. and Yen, G.C. (2006): Modulation of hepatic phase II phenol sulfotransferase and antioxidant status by phenolic acids in rats. J. Nutr. Biochem., 17, 561-569.

Yoshikawa, M., Sugimoto, S., Nakamura, S., Sakumae, H. and Matsuda, H. (2007): Medicinal flowers. XVI. New dammarane-type triterpene tetraglycosides and gastroprotective principles from flower buds of Panax ginseng. Chem. Pharm. Bull., 55, 1034-1038. 